\title{
Long-term evaluation of spontaneous release of epiretinal membrane and its possible pathogenesis
}

\author{
This article was published in the following Dove Press journal: \\ Clinical Ophthalmology \\ I September 2017 \\ Number of times this article has been viewed
}

\author{
Teruyo Kida' \\ Seita Morishita ${ }^{2}$ \\ Masanori Fukumoto' \\ Takaki Sato' \\ Hidehiro Oku' \\ Tsunehiko Ikeda' \\ 'Department of Ophthalmology, \\ Osaka Medical College, Takatsuki, \\ ${ }^{2}$ Department of Ophthalmology, \\ Osaka Kaisei Hospital, Osaka, Japan
}

Purpose: To investigate the characteristics in spontaneous release of epiretinal membrane (ERM) during watchful waiting and to introduce a possible mechanism of pathogenesis as a photo essay.

Methods: Records from all patients with ERM were obtained from Osaka Medical College Hospital from January 2001 to October 2012. Visual acuity (VA), fundus photo, and optical coherence tomography (OCT) were reviewed using the medical records. For statistical analysis, VA measured with a Landolt chart was converted to the logarithm of the minimum angle of resolution ( $\log M A R)$. To investigate the pathogenesis of ERM, tryptase activity in vitreous, which plays a role in tissue fibrosis and remodeling, was measured in patients that underwent a vitrectomy for ERM, macular hole, and proliferative diabetic retinopathy (PDR).

Results: ERM was observed in 604 patients and spontaneous release of the ERM was observed in 13 patients with 14 eyes (four males and nine females, aged 33-78 years). Among the 14 eyes, mean VA did not change significantly through the release of the ERMs $(0.17 \pm 0.18$ before and $0.24 \pm 0.40$ after release, $P=0.544$ ). Nine eyes showed posterior vitreous detachment or vitreomacular traction on OCT images and five eyes did not. ERM was released in five eyes with no accompanying vitreous traction by OCT during watchful waiting and seems to have peeled off by itself by contracting and rolling from the inferior side. Three eyes with deteriorated VA underwent vitrectomy due to macular hole or pseudomacular hole. Vitreal tryptase activity was significantly higher in patients with ERM compared to those with PDR $(P<0.05)$.

Conclusion: Fundus photos of ERM auto-peeling were taken during long-term follow-up. Spontaneous release of ERM is possibly involved in vitreous traction or membrane contraction. In addition, tryptase may be involved in the development and contraction of ERM.

Keywords: epiretinal membrane, auto-peeling, contraction, pathogenesis, tryptase, internal limiting membrane

Epiretinal membrane (ERM) is caused by a fibroglial proliferation on the surface of the retina. Patients with vitreomacular traction syndrome are treated with ocriplasmin; however, it usually has little effect on the treatment of ERM. ${ }^{1,2}$ Whereas, some patients do show auto-peeling and spontaneous release of ERM without posterior vitreous detachment during watchful waiting, which involves the contraction of the ERM (Figures 1 and 2). However, this phenomenon usually takes a long time. Therefore, to investigate the characteristics in spontaneous release of ERM during watchful waiting, visual acuity (VA), fundus photo, and optical coherence tomography (OCT) were reviewed using our medical records obtained from Osaka Medical College Hospital from January 2001 to October 2012. Patient consent to review their medical records was not required by the institutional review board, and patient
Correspondence: Teruyo Kida

Department of Ophthalmology,

Osaka Medical College, 2-7

Daigaku-machi, Takatsuki,

Osaka 569-8686, Japan

Tel +81726831221

Fax +81726818195

Email opt038@osaka-med.ac.jp
Clinical Ophthalmology 2017:1। 1607-1610

1607

Dovepress in

http://dx.doi.org/10.214710PTH.S146692 (c) (1) @ 2017 Kida et al. This work is published and licensed by Dove Medical Press Limited. The full terms of this license are available at https://www.dovepress.com/terms.php hereby accept the Terms. Non-commercial uses of the work are permitted without any further permission from Dove Medical Press Limited, provided the work is properly attributed. For permission for commercial use of this work, please see paragraphs 4.2 and 5 of our Terms (https://www.dovepress.com/terms.php). 

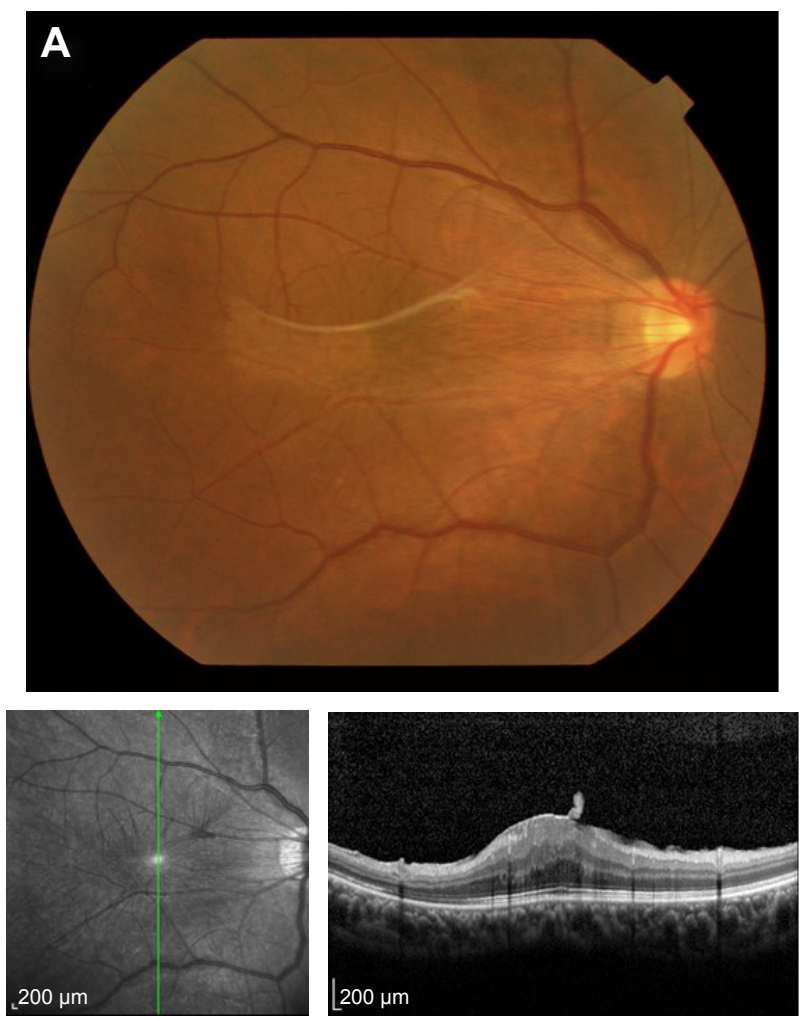
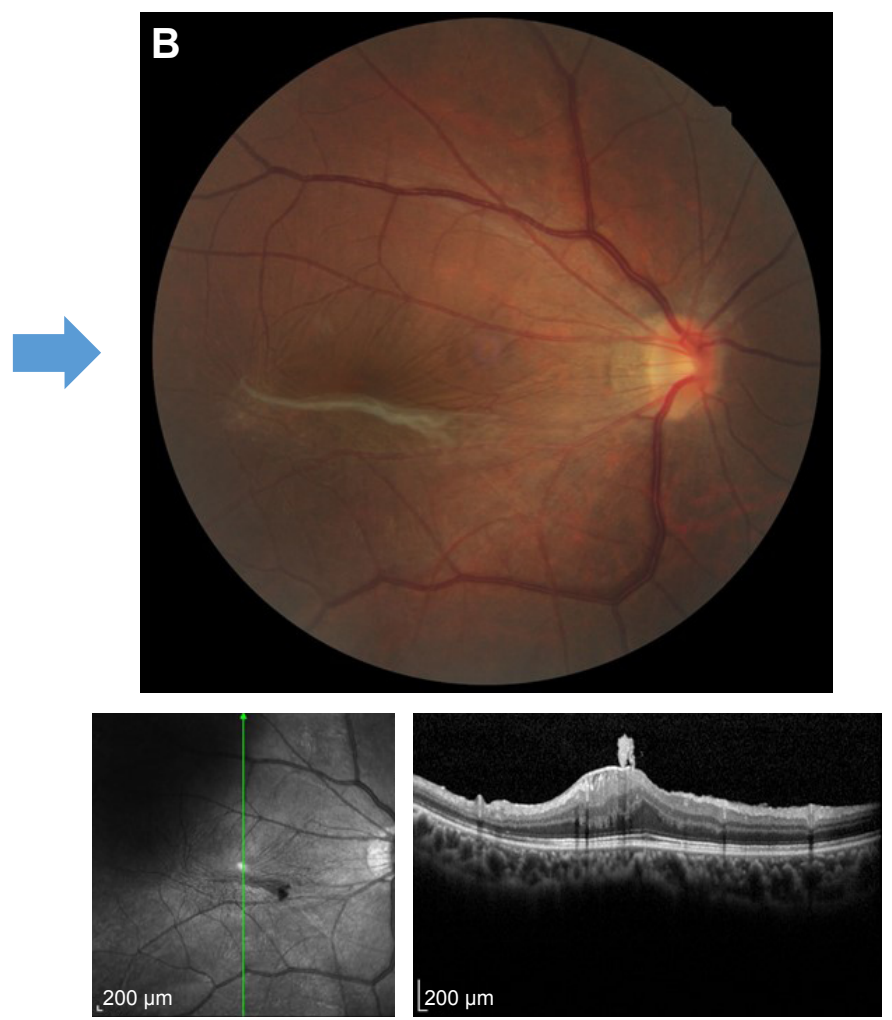

Figure I Auto-peeling of idiopathic ERM can be seen in these fundus photos and macular OCT from a 33-year-old female with idiopathic ERM. These photos and OCT were taken in (A) July 2010 and (B) October 2012. Visual acuity was improved from 10/20 to 14/20.

Abbreviations: ERM, epiretinal membrane; OCT, optical coherence tomography.

data were covered confidentially. For statistical analysis, VA measured with a Landolt chart was converted to the logarithm of the minimum angle of resolution (logMAR). ERM was observed in 604 patients and spontaneous release of ERM was observed in 13 patients with 14 eyes (four males and nine females, aged 33-78 years). Among the 14 eyes, mean VA did not change significantly through release of the ERMs $(0.17 \pm 0.18$ before and $0.24 \pm 0.40$ after release, $P=0.544$ ). In five eyes, the released ERM seemed to have peeled off by itself by contracting and rolling from the inferior side without accompanying vitreous traction by OCT during watchful waiting. Three eyes with deteriorated VA underwent vitrectomy due to macular hole or pseudomacular hole.
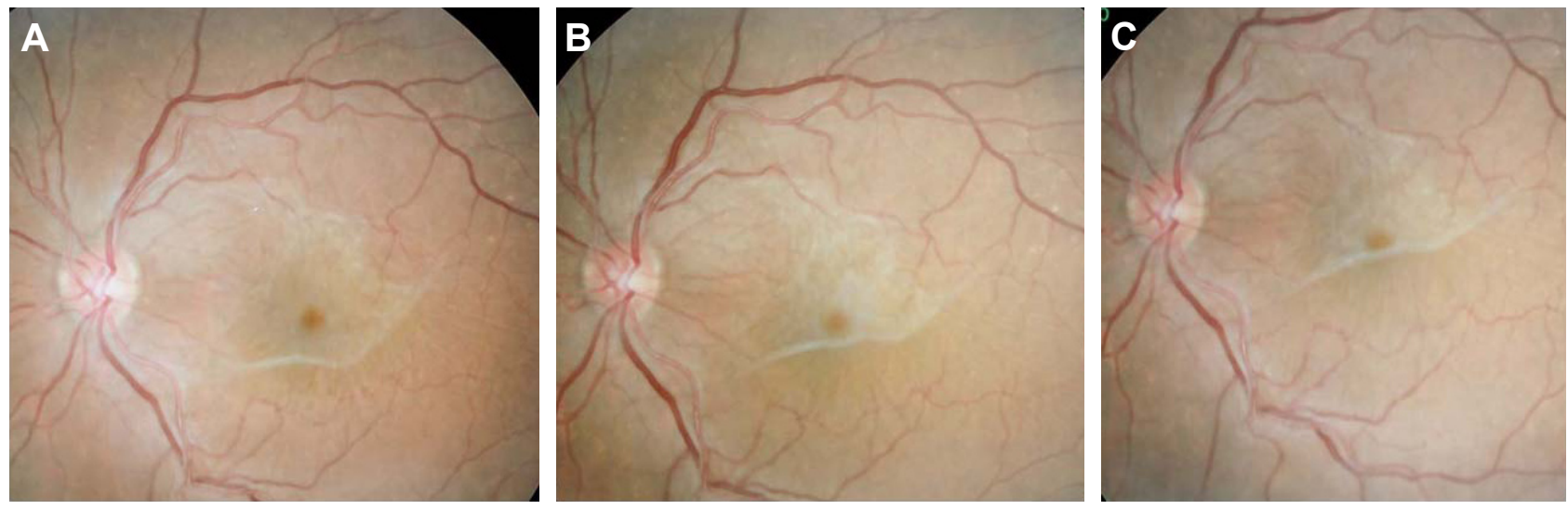

Figure 2 Auto-peeling of idiopathic ERM can be seen in these fundus photos from a 76-year-old female with idiopathic ERM. These photos were taken in (A) August 2008, (B) November 2009, and (C) February 2012

Abbreviation: ERM, epiretinal membrane. 

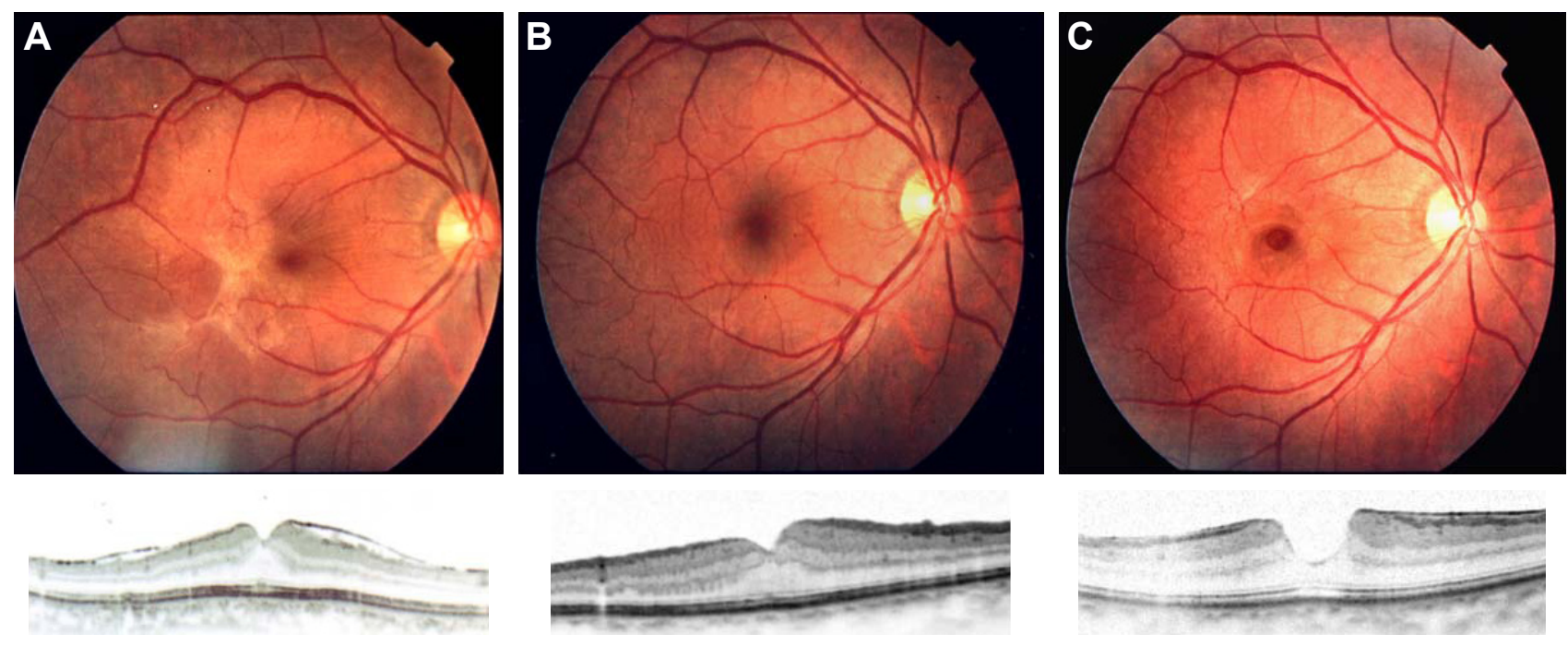

Figure 3 Recurrence of ERM in the macula after successful removal of idiopathic ERM by vitrectomy. Vitrectomy for idiopathic ERM was performed and visual acuity improved from 20/30 (A) to 20/20 (B). However, it reoccurred I year after the vitrectomy and the visual acuity decreased to 20/30 (C).

Abbreviation: ERM, epiretinal membrane.

Although interesting papers have been published regarding the pathohistology of ERMs ${ }^{3,4}$ and the remodeling of the extracellular matrix at the vitreoretinal interface, ${ }^{2}$ the pathomechanisms leading to ERM still remains unclear, which raises the following questions:

1. Why does ERM occur in the macula of healthy subjects?

2. Why does ERM sometimes show auto-peeling and spontaneous release without posterior vitreous detachment?

3. What causes the recurrence of ERM in the macula again after successful removal of idiopathic ERM by vitrectomy? (Figure 3)

4. Why does ERM reoccur despite the spontaneous release of ERM?

Based on the recurrent ERM depicted in Figure 3, it is also necessary to think about the presence of internal limiting membrane (ILM). The removal of ILM is the key for ERM recurrence, and ILM peeling in vitrectomy of ERM could result in a significantly lower ERM recurrence rate by preventing reproliferation and ensuring complete removal of ERM fragments. ${ }^{5}$ In addition, ERM tears and scrolling of tear edges include torn ILM (ILM ripping) histologically, ${ }^{6,7}$ and the presence of a postoperative ERM edge was recently reported to be associated with a higher risk of ERM recurrence. ${ }^{8}$

We add an interesting finding that may answer these questions by showing the possible involvement of tryptase in the development of idiopathic ERM (Figure 4). Tryptase activity in the vitreous was significantly higher in patients with ERM $(n=14)$ compared to those with PDR $(n=13)(P<0.05)$. The
Ethics Committee of Osaka Medical College, Takatsuki City, Osaka, Japan, approved this study. Tryptase is a serine protease produced in mast cells. Mast cells are located in the eye such as in choroid, ciliary body, conjunctiva, and sclera and play a role in tissue fibrosis, apoptosis, and remodeling. We have previously reported that tryptase activity in the vitreous increased in idiopathic ERM. ${ }^{9}$ It has also been reported that ERM shows immunoreactivity for collagen types 1 and 2 histopathologically, ${ }^{10}$ and tryptase stimulates fibroblast-mediated contraction of collagen type 1 gels, ${ }^{11}$ Thus, the pathogenesis of ERM is possibly related to ERM auto-peeling by contracting the ERM, which results in spontaneous release of ERM. Here, we would like to add the possibility that tryptase affects the development of ERM and contraction of ERM, and mast cells play a key role in the pharmacological treatment of

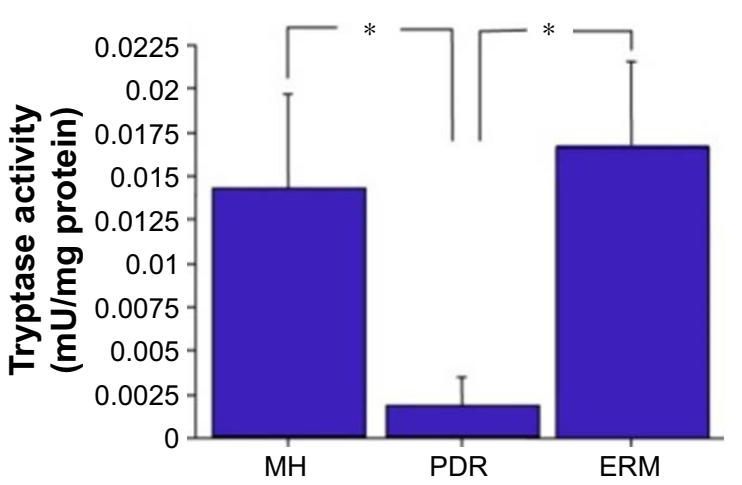

Figure 4 Tryptase activity in the vitreous. Tryptase activity in the vitreous was significantly higher in patients with ERM $(n=14)$ and $M H(n=14)$ compared to PDR $(\mathrm{n}=13) ; * \mathrm{p}<0.05$.

Abbreviations: ERM, epiretinal membrane; $\mathrm{MH}$, macular hole; PDR, proliferative diabetic retinopathy. 
ERM, although further research is needed to determine where tryptase in the vitreous comes from exactly.

\section{Disclosure}

The authors report no conflicts of interest in this work.

\section{References}

1. Spaide RF. Vitreomacular traction syndrome. Retina. 2012;32(Suppl 2): S187-S190; discussion S190-S183.

2. Bu SC, Kuijer R, Li XR, Hooymans JM, Los LI. Idiopathic epiretinal membrane. Retina. 2014;34(12):2317-2335.

3. Hiscott PS, Grierson I, McLeod D. Natural history of fibrocellular epiretinal membranes: a quantitative, autoradiographic, and immunohistochemical study. Br J Ophthalmol. 1985;69(11):810-823.

4. Kampik A. Pathology of epiretinal membrane, idiopathic macular hole, and vitreomacular traction syndrome. Retina. 2012;32(Suppl 2): S194-S198; discussion S198-S199.

5. Schechet SA, DeVience E, Thompson JT. The effect of internal limiting membrane peeling on idiopathic epiretinal membrane surgery, with a review of the literature. Retina. 2017;37(5):873-880.
6. Bovey EH, Uffer S. Tearing and folding of the retinal internal limiting membrane associated with macular epiretinal membrane. Retina. 2008; 28(3):433-440.

7. Sigler EJ, Randolph JC, Calzada JI. Incidence, morphology, and classification of epimacular membrane rip. Retina. 2013;33(6):1158-1165.

8. Gaber R, You QS, Muftuoglu IK, et al. Characteristics of epiretinal membrane remnant edge by optical coherence tomography after pars plana vitrectomy. Retina. Epub 2017 Jan 11. doi:10.1097/IAE. 0000000000001466.

9. Ikeda T, Nakamura K, Oku H, et al. The role of tryptase and anti-type II collagen antibodies in the pathogenesis of idiopathic epiretinal membranes. Clin Ophthalmol. 2015;9:1181-1186.

10. Kritzenberger M, Junglas B, Framme C, et al. Different collagen types define two types of idiopathic epiretinal membranes. Histopathology. 2011;58(6):953-965.

11. Skold CM, Ohkuni Y, Liu XD, Numerof R, Rennard SI. Co-cultured human mast cells stimulate fibroblast-mediated contraction of collagen gels. Inflammation. 2001;25(1):47-51.
Clinical Ophthalmology

\section{Publish your work in this journal}

Clinical Ophthalmology is an international, peer-reviewed journal covering all subspecialties within ophthalmology. Key topics include: Optometry; Visual science; Pharmacology and drug therapy in eye diseases; Basic Sciences; Primary and Secondary eye care; Patient Safety and Quality of Care Improvements. This journal is indexed on

Submit your manuscript here: http://www.dovepress.com/clinical-ophthalmology-journal

\section{Dovepress}

PubMed Central and CAS, and is the official journal of The Society of Clinical Ophthalmology (SCO). The manuscript management system is completely online and includes a very quick and fair peer-review system, which is all easy to use. Visit http://www.dovepress.com/ testimonials.php to read real quotes from published authors. 